\title{
Extended emission and spectral break in Cen A
}

\author{
Volker Beckmann* \\ François Arago Centre, APC, Université Paris Diderot, CNRS/IN2P3, CEA/DSM, Observatoire \\ de Paris, 13 rue Watt, 75205 Paris Cedex 13, France \\ E-mail: beckmanneapc.univ-paris7.fr
}

Pierre Jean

Centre d'Étude Spatiale des Rayonnements (CESR), OMP, UPS, CNRS; B.P. 44346, 31028

Toulouse Cedex 4, France

\section{Piotr Lubiński}

Centrum Astronomiczne im. M. Kopernika, Rabiańska 8, PL-87-100 Toruń, Poland

\section{Simona Soldi}

Laboratoire AIM - CNRS - CEA/DSM - Université Paris Diderot (UMR 7158), CEA Saclay, DSM/IRFU/SAp, 91191 Gif-sur-Yvette, France

\section{Regis Terrier}

APC, Université Paris Diderot, CNRS/IN2P3, CEA/DSM, Observatoire de Paris, 75013 Paris

The radio galaxy Cen $\mathrm{A}$ has been detected all the way up to the TeV energy range. This raises the question about the dominant emission mechanisms in the high-energy domain. Spectral analysis allows us to put constraints on the possible emission processes. Here we study the hard X-ray emission, in order to distinguish between a thermal or non-thermal inverse Compton process. The hard X-ray spectrum of Cen A shows a significant cut-off at energies $E_{C}=434_{-74}^{+109} \mathrm{keV}$ with an underlying power law of photon index $\Gamma=1.73 \pm 0.02$. A more physical model of thermal Comptonisation (compPS) gives a plasma temperature of $k T_{e}=206 \pm 62 \mathrm{keV}$ within the optically thin corona with Compton parameter $y=0.42_{-0.06}^{+0.09}$. The reflection component is significant at the $3.6 \sigma$ level with $R=0.12_{-0.10}^{+0.09}$. Extending this model to the gamma-ray range shows that a different physical component is responsible for the high-energy emission. The analysis of the SPI data provides no sign of significant emission from the radio lobes and gives a $3 \sigma$ upper limit of $f_{40-1000 \mathrm{keV}}<1.1 \times 10^{-3} \mathrm{ph} \mathrm{cm}^{-2} \mathrm{~s}^{-1}$. We find though some indication of emission near to the northern lobe of Cen A which we investigate in more detail.

8th INTEGRAL Workshop "The Restless Gamma-ray Universe"

September 27-30 2010

Dublin Castle, Dublin, Ireland

\footnotetext{
*Speaker.
} 


\section{Introduction}

The radio galaxy Centaurus A (NGC 5128) is probably the best studied active galactic nucleus (AGN). With a redshift of $z=0.001825$ equivalent to a distance of $d \sim 3.5 \mathrm{Mpc}$ the object is the brightest AGN at hard X-rays with $f_{20-100 \mathrm{keV}} \simeq 6 \times 10^{10} \mathrm{erg} \mathrm{cm}^{-2} \mathrm{~s}^{-1}$ [1]. For a review on Cen A, see [2]. Early hard X-ray observations by Ginga and balloon borne detectors indicated a power law slope of $\Gamma \sim 1.8$ and a possible break at $\sim 180 \mathrm{keV}$ [3]. The Compton Gamma-Ray Observatory's (CGRO; [4]) OSSE and COMPTEL instruments derived a more complex structure with a two-fold broken power law, with breaks at $E_{1}=150_{-20}^{+30} \mathrm{keV}$ and at $E_{2}=17_{-16}^{+28} \mathrm{MeV}$ [5]. More recent $R X T E$ and INTEGRAL observations showed an absorbed un-broken power law with photon index $\Gamma \simeq 1.8$ and an intrinsic hydrogen column density of $N_{\mathrm{H}} \simeq 10^{23} \mathrm{~cm}^{-2}[6,7]$. Nevertheless, Cen A shows a steeper spectral slope in the $\mathrm{MeV}$ range $\left(\Gamma_{\gamma}=2.3 \pm 0.1\right.$, [8] $)$ than in the hard $\mathrm{X}$-rays $\left(\Gamma_{X} \ll 2\right)$, thus a turnover has to occur somewhere between $100 \mathrm{keV}$ and a few $\mathrm{MeV}$ [9]. The source was also the only non-blazar AGN detected by CGRO/EGRET [10], showing again a steepening of the spectrum with $\Gamma_{0.03-10 \mathrm{GeV}}=2.4 \pm 0.3$ [11], consistent with the recent observations by Fermi/LAT $(\Gamma=$ $2.7 \pm 0.1,[12])$ and extending up to the $\mathrm{TeV}$ range, as seen by the $H E S S$ experiment $\left(\Gamma_{E>100 \mathrm{GeV}}=\right.$ $2.7 \pm 0.5,[13])$. Fermi/LAT observations also discovered extented gamma-ray emission which co-incides with the radio lobes [14].

The multi-year database of the INTEGRAL mission allows now to search the hard X-ray spectrum of Cen A for the expected turnover. In addition, we can test whether the emission model applicable for the X-ray domain can also explain the emission at gamma-rays.

\section{Data analysis}

In this study we use all INTEGRAL data on Cen A taken between March 3, 2003 and February 21,2009 , i.e. all observations dedicated to this radio galaxy. We used data of the imager IBIS/ISGRI in the 20-1000 keV band, IBIS/PICsIT data at $234-632 \mathrm{keV}$, and those of the spectrometre SPI between $40 \mathrm{keV}$ and $1850 \mathrm{keV}$. The two JEM-X monitors provide spectral information in the $3-30 \mathrm{keV}$ band. We selected the data up to an off-axis angle of $10^{\circ}$ for IBIS/ISGRI and SPI, and within $3^{\circ}$ for JEM-X. Data reduction was performed using the Offline Scientific Analysis (OSA) package version 9.0. The PICsIT data analysis followed the procedure described in Lubinski et al. [15]. The different instruments were not always switched on simultaneously. The effective exposure times are therefore different, with $145 \mathrm{ks}$ for JEM-X1, $96 \mathrm{ks} \mathrm{JEM-X2,} \mathrm{1,425} \mathrm{ks}$ IBIS/ISGRI, 2,076 ks IBIS/PICsIT, and 1,858 ks for SPI.

Errors quoted in this work are at the $3 \sigma$ level.

\section{The hard X-ray spectrum}

For spectral analysis we added all INTEGRAL data together, i.e. JEM-X1, JEM-X2, SPI, IBIS/PICsIT, and IBIS/ISGRI. A simple absorbed power law $(\Gamma=1.85 \pm 0.01)$ does not provide a good representation of the data $\left(\chi_{v}^{2}=1.83,104\right.$ d.o.f.). An absorbed cut-off power law model gives $\chi_{v}^{2}=1.07$ (103 d.o.f.) with a photon index of $\Gamma=1.73 \pm 0.02$ and $E_{C}=434_{-74}^{+109} \mathrm{keV}$. The spectral shape and the cut-off energy are not independent variables. A flatter spectrum is compensated in 
Table 1: Spectral fits to combined INTEGRAL data. All errors are at the $3 \sigma$ level.

\begin{tabular}{cccccc}
\hline \hline model & $\Gamma$ & $\begin{array}{c}E_{C} \text { or } k T_{e} \\
{[\mathrm{keV}]}\end{array}$ & $\mathrm{y}$ & $\mathrm{R}$ & $\chi_{v}^{2}$ (d.o.f.) \\
\hline power & $1.85 \pm 0.01$ & - & - & - & $1.83(104)$ \\
cut-off & $1.73 \pm 0.02$ & $434_{-74}^{+109}$ & - & - & $1.07(103)$ \\
pexrav & $1.75 \pm 0.04$ & $549_{-168}^{+387}$ & - & $0.07_{-0.07}^{+0.11}$ & $1.07(102)$ \\
compPS & - & $206 \pm 62$ & $0.42_{-0.06}^{+0.09}$ & $0.12_{-0.10}^{+0.08}$ & $1.02(101)$ \\
\hline
\end{tabular}

the fit by a lower cut-off energy. Nevertheless, a cut-off energy below $300 \mathrm{keV}$ and above 700 $\mathrm{keV}$ can be ruled out at a $99.7 \%$ confidence level. The spectral slope is better constrained by the data spanning $3-1000 \mathrm{keV}$. Even when considering different cut-off energies, the $3 \sigma$ range for the photon index is $1.68<\Gamma<1.78$.

The cut-off power law is a phenomenological description of the spectrum. A physical model should include the processes of thermal Comptonization. Here we apply the compPS model [16]. We assume that the plasma of temperature $T_{e}$ forms an infinite slab ontop of the accretion disk, with the plasma having an optical depth $\tau$. Since $T_{e}$ and $\tau$ are intrinsically strongly anticorrelated, we fit the Compton parameter $y=4 \tau k T_{e} /\left(m_{e} c^{2}\right)$ (with $m_{e}$ being the electron mass) instead of the optical depth. The temperature of the seed photons is not well constrained by the hard X-ray spectrum used here, thus we assume a multicolor disk with a fixed inner temperature $T_{b b}=10 \mathrm{eV}$.

The compPS model gives an electron plasma temperature of $k T_{e}=206 \pm 62 \mathrm{keV}$, and a Compton parameter $y=0.42_{-0.06}^{+0.09}$ corresponding to an optical depth of $\tau=0.26\left(\chi_{v}^{2}=1.02\right.$ for 101 d.o.f.). In this fit, we had to let the plasma temperature of the PICsIT spectrum to be independent from the other instruments, as the PICsIT spectrum appears slightly harder. This results for the PICsIT spectrum in a temperature as high as $675 \mathrm{keV}$, which cannot be constrained by the fit. The reflection strength is $R=0.12_{-0.10}^{+0.08}$, inconsistent with no reflection at the $3.6 \sigma$ level. Similar to the tight connection between the cut-off energy and the underlying spectral slope in the case of the cut-off power law model, in the compPS model the plasma temperature $T_{e}$ and the Compton parameter $y$ are linked to each other. Considering all combinations, the plasma temperature range is very wide $\left(300 \mathrm{keV}>\mathrm{kT}_{\mathrm{e}}>100 \mathrm{keV}\right)$ with a Compton parameter $0.34<y<0.57$ corresponding to a range of optical depth $0.14<\tau<0.69$.

The average model flux in the X-rays as detectable by INTEGRAL is $f_{(3-1000 \mathrm{keV})}=3 \times$ $10^{-9} \mathrm{erg} \mathrm{cm}^{-2} \mathrm{~s}^{-1}$, and thus Cen A displays a luminosity of $L_{X}=2.2 \times 10^{43} \mathrm{erg} \mathrm{s}^{-1}$.

For comparison we applied the more simple pexrav model which describes an exponentially cut-off power law spectrum reflected from neutral material, with the output spectrum being the sum of the cut-off power law and the reflection component [17]. In this case the best fit results $\left(\chi_{v}^{2}=1.07\right.$ for 102 d.o.f.) are a photon index of $\Gamma=1.75 \pm 0.04$, cut-off energy $E_{C}=549_{-168}^{+387} \mathrm{keV}$ and relative reflection of $R=0.07_{-0.07}^{+0.11}$, consistent with no reflection.

All the fit results concerning the combined INTEGRAL spectrum are summarized in Table 1.

\section{Extended emission}

As a radio galaxy, $\mathrm{Cen} \mathrm{A}\left(\mathrm{RA}=201.3651^{\circ}, \mathrm{DEC}=-43.0191^{\circ}\right)$ is known to have radio 


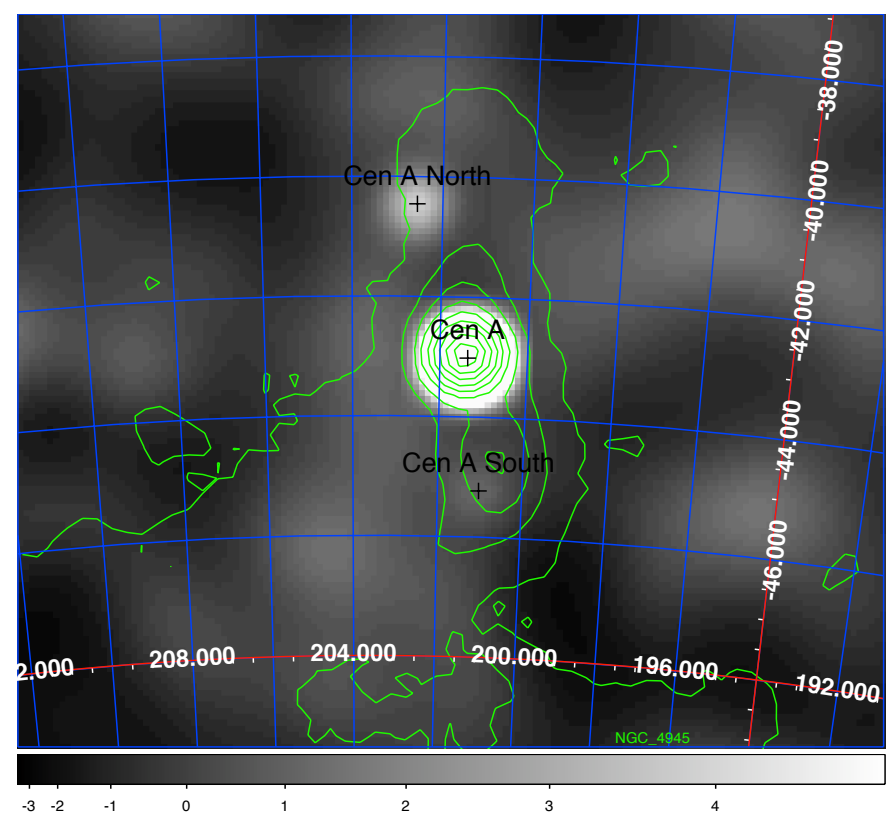

Figure 1: INTEGRAL/SPI significance map in the $40-1000 \mathrm{keV}$ energy band of the Cen A region. Overlaid are the contours of the WMAP radio map. Note that the hard X-ray emission of the lobes is not significant.

lobes extending out from the core by about $300 \mathrm{kpc}$. These lobes are located in the north at $\mathrm{RA}=201.5^{\circ}, \mathrm{DEC}=-39.8^{\circ}$ and in the south at $\mathrm{RA}=200.625^{\circ}, \mathrm{DEC}=-44.716^{\circ}$. Recently, Fermi/LAT detected emission from positions consistent with these lobes at significance levels of $5 \sigma$ (1FGL J1333.4-4036) and 8 $\sigma$ (1FGL J1322.0-4545) for the northern and southern lobes, respectively [14]. Here we use the data of the spectrometre SPI to test for emission of the lobes at hardest X-rays. Because of its large field of view and a resolution of 2.5. SPI is able to map large scale structures on the hard X-ray background. The spectra of the radio lobes and Cen A were extracted by model fitting, assuming that the sky intensity distribution consist of these 3 point sources, at the positions mentioned above. We performed the analysis in $10 \mathrm{keV}$ wide energy bins, covering the $40-1850 \mathrm{keV}$ range. Each energy bin is adjusted to the data for each germanium detector separately, assuming that the count rate is due to the sum of the sky contributions and the instrumental background. The latter is assumed to be proportional to the rate of saturating events in germanium detectors [18]. The spectra were rebinned in logarithmic spaced energy bins for the spectral analysis presented in Section 3. The analysis of the SPI data shows no sign of significant emission from the radio lobes in the south $\left(3 \sigma\right.$ upper limit $\left.f_{40-1000 \mathrm{kev}}<1.1 \times 10^{-3} \mathrm{ph} \mathrm{cm}^{-2} \mathrm{~s}^{-1}\right)$ and in the north with $f=1.0 \pm 0.4 \times 10^{-3} \mathrm{ph} \mathrm{cm}^{-2} \mathrm{~s}^{-1}$ for a non-significant signal on the $2.5 \mathrm{\sigma}$ level.

We investigated the excess in the north of Cen A further, by fitting a source model in various energy bands to the northern lobe in which position, extension, and orientation angle of a two-dimensional asymmetric Gaussian were left free to vary. In this case we find a excess of $f_{80-400 \mathrm{keV}}=(9.1 \pm 7.8) \times 10^{-4} \mathrm{phcm}^{-2} \mathrm{~s}^{-1}(3 \sigma$ error $)$ at $\mathrm{RA}=202.9^{\circ}, \mathrm{DEC}=-40.2^{\circ}$. This is at $1.1^{\circ}$ distance from the radio and $1.6^{\circ}$ from the Fermi/LAT position. In addition, we extracted a spectrum from the best fit position of the northern lobe. The result is shown in Fig. 2 


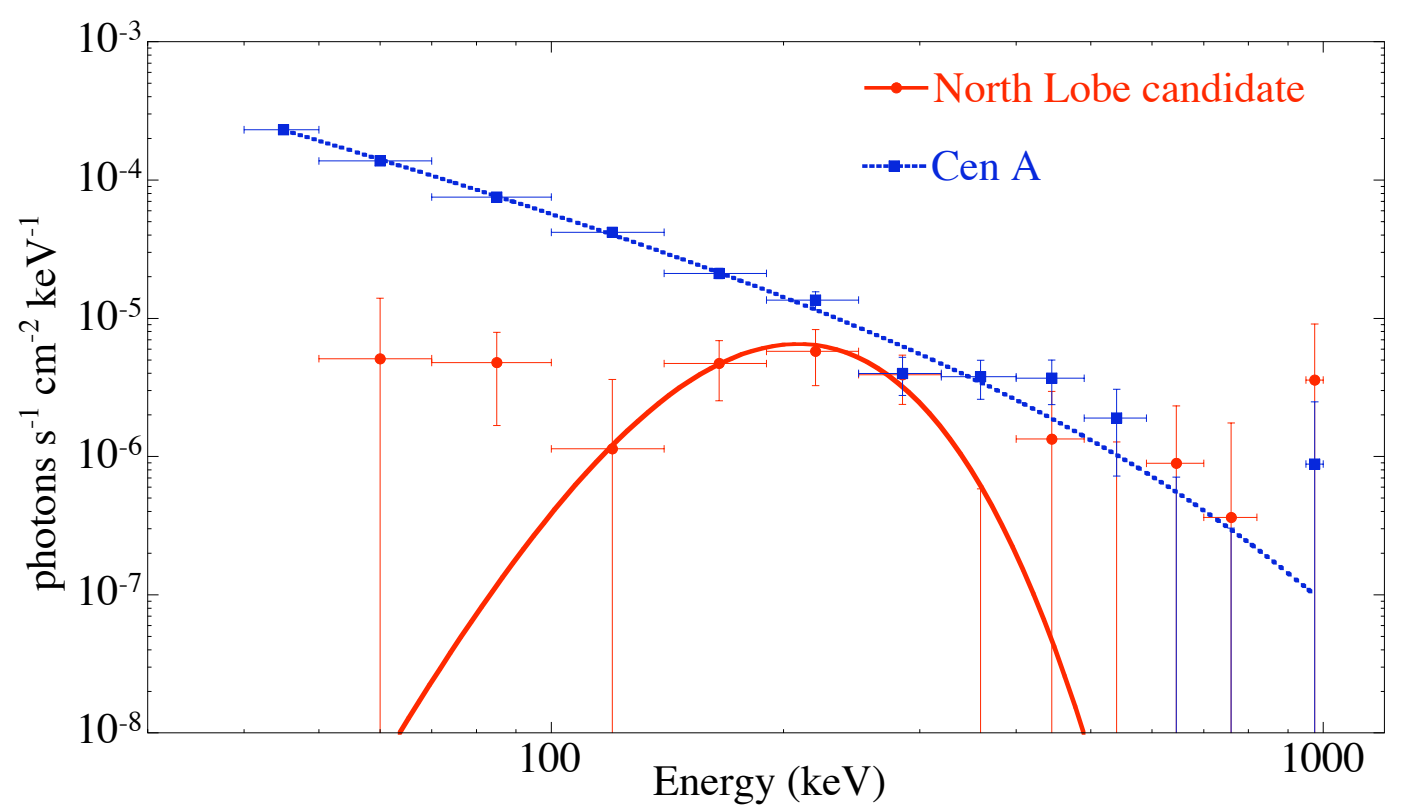

Figure 2: Spectrum of Cen A (blue) and of the north lobe candidate (red) based on the INTEGRAL/SPI data.

together with the spectrum of Cen A. It shows that the excess is sustained by three energy bins between $140 \mathrm{keV}$ and $320 \mathrm{keV}$, with significances larger than $2 \sigma$ and a flux of $f_{140-320 \mathrm{keV}}=$ $(9 \pm 2) \times 10^{-4} \mathrm{phcm}^{-2} \mathrm{~s}^{-1}(\sim 4 \sigma)$. The total flux in the feature between $40-1000 \mathrm{keV}$ is $1.40 \pm$ $0.5 \times 10^{-3} \mathrm{ph} \mathrm{cm}^{-2} \mathrm{~s}^{-1}(2.6 \sigma)$ which is compatible with the flux obtained assuming a point source, i.e. $(0.9 \pm 0.5) \times 10^{-3} \mathrm{phcm}^{-2} \mathrm{~s}^{-1}$. It has to be noted that compared to the point source hypothesis, the flux in Cen A is reduced from $(1.28 \pm 0.05) \times 10^{-2} \mathrm{phcm}^{-2} \mathrm{~s}^{-1}$, assuming that the north lobe candidate is a point source, to $(1.19 \pm 0.04) \times 10^{-2} \mathrm{ph} \mathrm{cm}^{-2} \mathrm{~s}^{-1}$, when an extended feature is assumed for the northern lobe candidate.

The $3.5 \sigma$ signal is too low in order to claim a significant detection at this point. Even if we consider this flux to be a reliable measurement, the hard X-ray lobe emission of Cen A can still be lower, as also other sources in the field can contribute, e.g. in the north 1FGL J1307.0-4030 detected by Fermi/LAT, or another so far undetected blazar in the background. Based on the SPI data, it is not yet possible to determine whether the excess we detect is extended or resulting from a point-like source.

If we consider the flux value of $f=10^{-3} \mathrm{ph} \mathrm{cm}^{-2} \mathrm{~s}^{-1}$ an upper limit, it agrees with the expected energy distribution presented by [14]. At an energy of $300 \mathrm{keV}$, they expect a contribution to the SED of $\sim 5 \times 10^{12} \mathrm{Jy} \mathrm{Hz}$ in the case of the southern lobe, whereas the upper limit based on the SPI data corresponds to $<10^{13} \mathrm{Jy} \mathrm{Hz}$. In the case of the northern lobe, the emission expected from the Fermi/LAT extrapolation is even lower. Therefore, unless about five times more SPI data will be accumulated on Cen A, the lobes will most likely escape detection at hardest X-rays.

\section{Discussion and Conclusion}

In the work presented here the Cen A spectrum shows a reflection component when applying a Compton reflection model (compPS). Although the reflection strength is comparably low $(R=$ 
$\left.0.12_{-0.10}^{+0.08}\right)$, it is inconsistent with no reflection on the $3.6 \sigma$ level. The plasma temperature is $k T_{e}=$ $206 \pm 62 \mathrm{keV}$ with a Compton parameter $y=0.42_{-0.06}^{+0.09}$.

Extrapolating the cut-off power law model, the compPS or the pexrav model into the Fermi/LAT energy range shows that the inverse Compton component dominating the X-rays cannot be responsible for the gamma-ray emission. Both models predict no detectable flux at energies above $100 \mathrm{MeV}$. Thus, an additional component, e.g. non-thermal (jet) emission, has to come into play here. This is a well established fact (e.g. [19], [12]) but the perfect match of the Comptonisation model to the INTEGRAL data rules out the possibility that the dominant emission in the X-rays is non-thermal, i.e. also arising from the jet as the gamma-ray emission [20]. This is also indicated by the iron line and the Compton reflection hump. The Fermi collaboration presented already a model considering the combined thermal inverse Compton plus a non-thermal gamma-ray component to explain the X-ray to VHE emission, although they could not rule out that the X-rays require a non-thermal modeling [12].

The extended emission we see at a low significance level in the INTEGRAL/SPI data and which coincides with the northern lobe of Cen A, cannot be detected beyond doubt. If this detection is not an artifact, there are several possibilities for its origin. It can be connected to the emission detected by Fermi/LAT, although one would expect a stronger emission from the southern lobe in this case. Another possibility is the detection of a background blazar in the hard X-rays. As this source would be a point source, it should show up though also in the hard X-ray data of IBIS/ISGRI, which is not the case. It might well be that this hard X-ray feature cannot be verified before the advent of a new generation of $\mathrm{MeV}$ telescopes, such as Compton telescopes like CAPSiTT, currently under study for ESA's M3 mission call.

\section{References}

[1] Beckmann, V., Soldi, S., Ricci, C., et al. 2009, A\&A, 505, 417

[2] Israel, F. P. 1998, A\&ARv, 8, 237

[3] Miyazaki, S., Takahashi, T., Gunji, S., et al. 1996, PASJ, 48, 801

[4] Gehrels, N., Chipman, E. \& Kniffen, D. A. 1993, A\&AS, 97, 5

[5] Steinle, H., Bennett, K., Bloemen, H., et al. 1998, A\&A, 330, 97

[6] Rothschild, R. E., Wilms, J., Tomsick, J., et al. 2006, ApJ, 641, 801

[7] Soldi, S., Beckmann, V., Bassani, L., et al. 2005, A\&A, 444, 431

[8] Collmar, W., Bennett, K., Bloemen, H., et al. 1999, Astrophysical Letters Communications, 39, 57

[9] Steinle, H. 2010, PASA, 27, 431

[10] Hartman, R. C., et al. 1999, ApJS, 123, 79

[11] Sreekumar, P., Bertsch, D. L., Hartman, R. C., et al. 1999, Astroparticle Physics, 11, 221

[12] Abdo, A. A., et al. 2010, ApJ, 719, 1433

[13] Aharonian, F., et al. 2009, ApJ, 695, L40

[14] Abdo, A. A., et al. 2010, ApJ, 719, 1433 
[15] Lubiński, P. 2009, A\&A, 496, 557

[16] Poutanen, J., \& Svensson, R. 1996, ApJ, 470, 249

[17] Magdziarz, P., \& Zdziarski, A. A. 1995, MNRAS, 273, 837

[18] Jean, P., Vedrenne, G., Roques, J. P., et al. 2003, A\&A, 411, L107

[19] Johnson, W. N., Zdziarski, A. A., Madejski, G. M., et al. 1997, Proceedings of the Fourth Compton Symposium, 410, 283

[20] Beckmann, V., Jean, P., Lubiński, P., Soldi, S., \& Terrier, R. 2010, submitted to A\&A 